\title{
Lower expression of the RASSF10 gene induces myeloma cell proliferation due to hypermethylation of the gene promoter in multiple myeloma
}

\section{Jin Chen}

Tianjin Medical University General Hospital https://orcid.org/0000-0001-8352-3576

Hui Liu

Tianjin Medical University General Hospital

Zhaoyun Liu

Tianjin Medical University General Hospital

Qing Shao

Tianjin Medical University General Hospital

\section{Fengjuan Jiang}

Tianjin Medical University General Hospital

\section{Siyang Yan}

Tianjin Medical University General Hospital

\section{Lijuan Li}

Tianjin Medical University General Hospital

Jia Song

Tianjin Medical University General Hospital

\section{Kai Ding}

Tianjin Medical University General Hospital

Rong Fu ( $\boldsymbol{D}$ florai@sina.com )

https://orcid.org/0000-0002-9928-9224

\section{Primary research}

Keywords: Multiple myeloma, RASSF10, DNA methylation, Epigenetics, Tumor suppressor gene

Posted Date: June 29th, 2020

DOl: https://doi.org/10.21203/rs.3.rs-23701/v2

License: (1) (1) This work is licensed under a Creative Commons Attribution 4.0 International License.

Read Full License 


\section{Abstract}

Background: Multiple myeloma (MM) is an incurable malignant neoplasm of plasma cells, in which genetic defects, epigenetic aberrations and bone marrow microenvironment are involved in the pathogenesis. RASSF10 acts as a tumor suppressor gene by methylation in glioma and several other cancers, but its role in MM remains unknown.

Methods: In order to explore the role of RASSF10, mRNA expression was detected in MM patients and analyzed with overall survival.

Results: Expression of the RASSF10 gene significantly decreased in newly diagnosed MM patients, and was positively correlated with overall survival. RPMI-8226 and OPM-2 cell lines with lower RASSF10 expression were selected for further study. Overexpression of RASSF10 in these two cell lines inhibited proliferation and induced apoptosis. The RASSF10 gene promoter in MM cell lines was hypermethylated, and downregulated after decitabine treatment. Meanwhile, expression of the RASSF10 gene was upregulated. MM cells with overexpression of RASSF10 were injected into nude mice and exerted anti-MM activity in vivo.

Conclusions: Low expression of RASSF 10 contributed to the proliferation of myeloma cells by hypermethylation of its promoter.

\section{Background}

Multiple myeloma ( $\mathrm{MM})$ is a malignant neoplasm of plasma cells that accumulate in the bone marrow $(B M)$ and is characterized by end-organ damage (CRAB) caused by secretion of a monoclonal protein ( $M$ protein) (1). Over the last 10 years, therapy of MM has improved using novel agents, including proteasome inhibitors and immunomodulatory drugs, in combination with autologous stem cell transplantation, alkylating agents and/or glucocorticoids. Unfortunately, MM remains an incurable disorder and all patients ultimately relapse due to the development or drug resistance. Therefore, further breakthroughs are still needed to improve patient outcome (2). Apart from the central role of genetic defects, an increasing number of studies has shown that epigenetic aberrations are also involved in the pathogenesis of MM. DNA methylation and post-translational histone modifications are the most common epigenetic mechanisms known to disturb normal gene expression (3-6).

The RASSF family encodes distinct tumor suppressors, which consists of six classical members (RASSF1-6) and four N-terminal members (RASSF7-10). The former group contains both the RA domain and the SARAH domain, and the latter contains an $R A$ domain within its extreme $\mathrm{N}$ termini but lacks the $S A R A H$ domain. RASSF functions shown epigenetic silencing, $R A S$ signaling, microtubule stability, cell cycle apoptosis, and immune system and nuclear transport $(7,8)$. RASSF10, as the newest member, has a CpG island of 2254 bp with $209 \mathrm{CpGs}$ and acts as a tumor suppressor gene by methylation of the gene promoter in glioma and several other cancers, such as thyroid cancer, prostate carcinoma, and leukemia (9-12). Recent research shows that RASSF10 is hypermethylated in B-acute lymphocytic leukemia (B-ALL) 
and chronic Lymphocytic Leukemia (CLL), and associated with prognostic parameters (13). MM is a Bcell malignant disease, which has been investigated for treatment with DNA methyltransferase (DNMT) inhibitors (decitabine or 5-azacytidine) (14). However, the role of RASSF10 in MM remains unknown.

\section{Materials And Methods}

\section{Patients}

Thirty newly diagnosed MM patients, 17 remission patients and 19 normal controls were enrolled in this study. All the patients were inpatients in the Department of Hematology, Tianjin Medical University General Hospital from June 2016 to August 2017. All MM patients were diagnosed according to the Guidelines for the Diagnosis and Management of Multiple Myeloma in China (2017 revision) (15). The clinical features of all MM patients are shown in Table 1. The remission patients who received bortezomib-basic regimens for at least four cycles were defined as very good partial response, complete response or stringent complete responses. Meanwhile, supportive therapies were given in these patients, including blood transfusion and anti-infective agents. Ten milliliters of bone marrow (BM) was taken from the patients and normal controls. This study was approved by the Ethical Committee of the Tianjin Medical University (IRB2020-WZ-075 and IRB2020-DW-04). Written informed consent was obtained from the patients for publication.

\section{Cell lines}

Human MM cell lines RPMI-8226, OPM-2 and U266 were obtained from the Cell Culture Center, Institute of Basic Medical Sciences Chinese Academy of Medical Sciences. All cell lines were cultured in 90\% RPMI1640 supplemented with $10 \%$ fetal bovine serum (FBS) (Gibco, USA) at $37^{\circ} \mathrm{C}$ with $5 \% \mathrm{CO}_{2}$ and $95 \%$ humidity.

\section{Magnetic-activated cell sorting (MACS)}

BM mononuclear cells (BMMCs) were isolated from heparin-anticoagulated BM of $\mathrm{MM}$ and normal controls using Ficoll-Hypaque density gradient centrifugation. $\mathrm{CD}_{138^{+}}$cells were purified using the antiCD138 mAb-conjugated MicroBeads (Miltenyi Biotec, Bergisch Gladbach, Germany). Ten million cells were resuspended in $80 \mathrm{ml}$ buffer. Then, $20 \mathrm{ml}$ CD138 MicroBeads (Miltenyi Biotec) were added and incubated at $4^{\circ} \mathrm{C}$ in the dark for $15 \mathrm{~min}$. After washing with $2 \mathrm{ml}$ buffer, the cells were centrifuged at $300 \mathrm{~g}$ for $5 \mathrm{~min}$. The cells were resuspended up in $500 \mathrm{ml}$ buffer. The MS column was placed in the magnetic field of a suitable MACS separator (Miltenyi Biotec). After preparing the column by rinsing with 3' $500 \mathrm{ml}$ buffer, the cells were applied to the column. The column was washed with $1 \mathrm{ml}$ buffer and all flowthrough containing unlabeled cells was collected. Magnetically labeled cells were immediately flushed out by firmly pushing the plunger into the column and collected. The purity of enriched CD $138^{+}$cells isolated was evaluated by flow cytometry and was generally $>90 \%$.

\section{RNA expression analysis}


Total RNA was isolated using TRIzol (Invitrogen, Carlsbad, California, USA). First-strand cDNA was generated using the FastQuant RT Kit (Tiangen, Beijing, China). Quantitative real-time polymerase chain reaction (PCR) was performed in triplicate with SYBR Green (Tiangen) using IQ5 PCR instrumentation (Bio-Rad, Hercules, CA, USA). Quantitative real-time PCR primers were as follows: 5'-

GGCTCAACACGGACCTAGAG-3'(F), 5'-GTCAGCTCCAAAGTGTGCAA-3'(R). $\beta$-Actin was used as an internal control.

\section{DNA isolation and methylation analysis by bisulfite sequencing}

DNA was extracted from MM cell lines and normal controls with a TIANamp Genomic DNA kit (Tiangen), and concentrations of DNA were determined by a micro-ultraviolet spectrophotometer (Bio-Rad). Bisulfite sequencing was performed by Genechem (Shanghai, China). The experiment was repeated three times and the values were averaged.

\section{Lentivirus transfection}

RASSF10 lentivirus was purchased from Genechem, and lentivirus was transfected into MM cell lines and cells. The MOI=100, the cells were not selected using antibiotics, and Con238 (Ubi-MCS-3FLAG-SV40EGFP-IRES-puromycin) was used as a control. The efficiency of transfection was measured by flow cytometry and inverted microscopy.

\section{Cell proliferation}

MM cell lines and cells at $72 \mathrm{~h}$ after transfection were analyzed for proliferation and apoptosis. Cell proliferation was determined using a Cell Counting Kit-8 (CCK-8) (Engreen, Beijing, China). Absorbance at $450 \mathrm{~nm}$ was read at 1, 2, 3 and 4 days using a 96-well microplate reader (BioTek, Winooski, VT, USA). The experiment was repeated three times and the values were averaged.

\section{Flow cytometry}

The proportion of cells undergoing apoptosis was measured using the Apoptosis Detection kit (BD Bioscience, San Diego, CA, USA). Cells were stained with fluorescein isothiocyanate-annexin $V$ and propidium iodide (PI) and analyzed with a flow cytometer (FACScan; BD Biosciences, Mountain View, CA, USA). All assays were conducted in triplicate. The experiment was repeated three times and the values were averaged.

\section{Western blotting}

Cellular proteins were extracted in radio-immunoprecipitation assay buffer (Beyotime, Shanghai, China) and protein concentrations were determined using a BCA assay kit (Beyotime). Cell extracts (30 mg) were boiled with equal amounts of loading dye for 10 min and separated by $12 \%$ polyacrylamide gel electrophoresis and then transferred onto nitrocellulose membranes (Hybond-ECL; Thermo Fisher Scientific, Shanghai, China). Membranes were blocked in Phosphate buffered saline (PBS) with $0.1 \%$ 
Tween 20 (PBS-T) containing 5\% non-fat milk for $1 \mathrm{~h}$, and incubated with primary and secondary antibodies in PBS-T containing $5 \%$ non-fat milk. The following primary antibodies were used: RASSF10 (diluted 1:1000) (Abcam, Cambridge, UK), bcl-2 (diluted 1:1000), caspase3 (diluted 1:500), GAPDH (diluted 1:1000) (Cell Signaling Technology, Danvers, MA, USA). Primary antibody incubation was carried out overnight at $4{ }^{\circ} \mathrm{C}$. The membranes were washed with wash buffer ( $1 \times$ PBS and $0.01 \%$ Tween-20) and incubated with anti-rabbit or anti-mouse secondary antibody. The experiment was repeated three times and the values were averaged.

\section{In vivo tumor growth in nude mice}

Female BALB/c-nu nude mice aged 4-5 weeks were purchased from Beijing Hua Fukang Bioscience Company and were housed and monitored in a pathogen-free environment. RPMI-8226 MM cell line and transfected cells $\left(n=10^{7}\right)$ were prepared in $100 \mathrm{ml}$ serum-free RPMI- 1640 medium and injected subcutaneously into the right dorsal flank of nude mice ( $n=3$ each group). Measurement of tumor volume and tumor quality was performed after 21 days, and tumor volume $(\mathrm{V})$ was calculated using the formula: $V=0.5 \times a \times b^{2}$, where $a$ and $b$ represent the longer and shorter tumor diameters, respectively. At the end of each study, animals were killed and tumors were collected and fixed in formalin for hematoxylin and eosin (HE) staining and immunohistochemical staining of anti-CD138 or anti-RASSF10 antibody to assess tumor growth. CD138 and RASFF10 staining was quantified by Image $\mathrm{J}$ software. All procedures were approved by the Animal Ethics Committee of the Tianjin Medical University General Hospital.

\section{Statistical analysis}

Student's $t$-test was conducted for two-group comparisons. For many-group comparisons, one-way ANOVA (if the data were normally distributed) or Kruskal-Wallis test (if the data were not normally distributed) was used. The data are expressed as the mean \pm SEM or median. Kaplan-Meier survival curves were constructed, and difference in survival rates was tested by log-rank test. Statistical analyses were performed using SPSS version 21.0. A value of $p<0.05$ was considered significant.

\section{Results}

\section{Expression of the RASSF10 gene significantly decreased in newly diagnosed MM patients and MM cell} lines, and was positively correlated with overall survival

The purity of $\mathrm{CD} 138^{+}$cells sorted from BMMCs was $>90 \%$. Expression of RASSF 10 mRNA in the newly diagnosed MM (NDMM) group (0.20 \pm 0.29$)$ was significantly lower than that in the remission group $(0.64 \pm 0.61)$ and normal control group $(0.62 \pm 0.61)$ (both $p<0.01)$, while there was no significant difference between the latter two groups (Fig. 1A). Expression of RASSF10 mRNA in MM cell lines RPMI-8226, OPM2 and U266 was significantly more decreased than NCl-H929 and LP-1 (Fig. 1B). Protein expression of the RASSF10 gene was downregulated in the newly diagnosed MM group and MM cell lines RPMI-8226, OPM-2 and U266 (Fig. 1C). 
The median follow-up time of NDMM patients was 18 months ( $95 \% \mathrm{Cl} 15-22$ months). The overall survival of patients with higher expression of RASSF 10 mRNA (median selected as the cut-off value $=$ 0.1 ) was 21 months ( $95 \% \mathrm{Cl} 18-25$ months), which was significantly longer than for patients with lower expression of RASSF10 mRNA (13 months, 95\% Cl 9-17 months) ( $p<0.05)$ (Fig. 1D).

\section{Overexpression of RASSF10 in RPMI-8226 and OPM-2 cells inhibited proliferation and induced apoptosis}

The transfection efficiency rates of the RASSF10 gene in MM cell lines RPMI-8226, OPM-2 and U266 was $69.3 \%, 58.67 \%$ and $34.7 \%$, respectively. RPMI-8226 and OPM-2 cells were chosen for further experiments. After transfection, expression of the RASSF10 gene was significantly upregulated in RPMI-8226 and OPM2 cells ( $p=0.0007$ and $p=0.0185$, respectively) (Fig. $2 A)$.

Proliferation of RPMI-8226/RASSF10 cells by CCK-8 was significantly decreased compared with RPMI$8226 /$ control at 48,72 and $96 \mathrm{~h}(p<0.001, p<0.001$ and $p<0.001$, respectively), while there was no significant difference at $24 \mathrm{~h}$ (Table 2 and 3), (Fig. 2B1,2). The apoptosis rate of RPMI-8226/RASSF10 cells was elevated significantly at 24,72 and $96 \mathrm{~h}(p<0.05, p<0.05$ and $p<0.01$, respectively). Similar results were found for OPM-2/RASSF10 cells (Table 2, Fig. 2C1,2; D1,2). Furthermore, apoptosis-related proteins were detected by western blotting. Protein expression of the RASSF10 gene was upregulated. The expression of apoptosis-related protein the cleaved caspase-3 was up-regulated, while bcl-2, an apoptosis inhibitor, was down-regulated at the protein level. (Fig. 2E).

\section{The RASSF10 gene promoter in MM cell lines was hypermethylated and expression of the RASSF10 gene was upregulated after decitabine treatment}

Methylation of the RASSF10 gene promoter (CpG island divided into Pairs 1-6) in MM cell lines RPMI8226 and OPM-2 was detected by bisulfite sequencing. The methylation levels of Pair 2 in RPMI-8226 and Pairs 2 and 5 in OPM-2 cells were significantly higher compared with those in normal controls $(p<0.001, p<0.05$ and $p<0.05$, respectively), while other levels were low (Fig. $3 \mathrm{~A})$.

We treated these MM cell lines with decitabine $(0,0.3125,0.625,1.25,2.5,5,10$ or $20 \mathrm{mM})$ at 24,48 and $72 \mathrm{~h}$. Proliferation of RPMI-8226 cells significantly reduced from $0.9640(0 \mathrm{mM})$ to $0.8606(1.25 \mathrm{mM})$, $0.8567(2.5 \mathrm{mM}), 0.8430(5 \mathrm{mM}), 0.8103(10 \mathrm{mM})$ and $0.8263(20 \mathrm{mM})$ at $48 \mathrm{~h}$. The results were better at $72 \mathrm{~h}$, from $1.1340(0 \mathrm{mM})$ to 0.5297 (20 mM) (Fig. 3B1). Apoptosis of RPMI-8226 cells at $72 \mathrm{~h}$ significantly increased to $22 \%(1.25 \mathrm{mM}), 28 \%(2.5 \mathrm{mM}), 39 \%(5 \mathrm{mM}), 43 \%(10 \mathrm{mM})$ and $44 \%(20 \mathrm{mM})$ (Fig. 3B2). In OPM-2 cells, there were significant differences from $1.25 \mu \mathrm{M} \mathrm{DAC}$ at $72 \mathrm{~h}$, which are less effective than RPMI-8226 (Fig 3C1,2).

We further detected the methylation of the RASSF10 gene promoter in RPMI-8226 cells after decitabine treatment. The methylation level was partly downregulated in Pair 2 after $2.5 \mathrm{mM}$ decitabine treatment at $72 \mathrm{~h}(p<0.05)$ (Fig 3D). At the same time, the level of RASSF10 mRNA in RPMI-8226 cells significantly increased from $\left(1.57 \times 10^{-5} \pm 2.02 \times 10^{-6}\right)$ to $\left(1.44 \times 10^{-3} \pm 7.79 \times 10^{-4}\right)$ after decitabine treatment $(2.5 \mathrm{mM})$ at 
$48 \mathrm{~h}(p<0.05)$ (Fig 3E). The results indicated that the upregulated RASSF10 gene level was due to demethylation by decitabine.

\section{Overexpressed RASSF10 exerts anti-MM activity in vivo}

Nude mice (BALB/c-nu) were injected subcutaneously with RPMI-8226 and OPM-2 cells in the right scapular region. The RPMI-8226 nude mice had subcutaneous tumor formation. The lymph nodes, liver and spleen were significantly enlarged, and myeloma cells infiltrated the liver. After the recovery of RASSF10 expression (RPMI-8226/RASSF10 cells), the subcutaneous tumor formation volume was reduced, and myeloma cells did not infiltrate the liver. The tumor volume and mass of the RPMI-8226 control group were $96.97 \pm 15.22 \mathrm{~mm}^{3}$ and $87.57 \pm 19.75 \mathrm{mg}$ at 21 days, respectively. The tumor volume and mass of the RPMI-8226/RASSF10 group were significantly reduced to $16.56 \pm 3.15 \mathrm{~mm}^{3}$ and $19.90 \pm 4.60 \mathrm{mg}$, respectively (both $p<0.001$ ) (Fig. 4A, B).

The tumor tissue formed subcutaneously in nude mice was stained with HE and showed a large number of irregular cells, short spindle or cubic, with irregular nuclei, coarse staining, and obvious nucleoli. Immunohistochemistry showed $\mathrm{CD} 138^{+}$cells (40-50\%) in the RPMI-8226/control group, which became weaker ( 30\%) in the RPMI-8226/RASSF10 group. RASSF10 gene expression was upregulated from 10$20 \%$ in the RPMI-8226/control group to $50-60 \%$ in the RPMI-8226/RASSF10 group (Fig. 4C).

\section{Discussion}

RASSF10 acts as a tumor suppressor gene in some cancers, including non-hematological and hematological malignancies, such as ALL and CLL. However, the role of RASSF10 remains unknown in MM. In our study, we found that RASSF10 mRNA expression was significantly lower in newly diagnosed MM patients compared with normal controls, and upregulated after remission, which was associated with survival. Furthermore, overexpression of the RASSF10 gene inhibited proliferation of MM cell lines in vitro. After injection of RPMI-8226/RASSF10 cells into mice, we found that myeloma mass decreased. These results indicate that the RASSF10 gene may contribute to the pathogenesis of MM. However, the mechanism of this gene remains unclear in MM.

Wei et al. (16) studied the function of RASSF10 in gastric cancer, and showed that the RASSF10 gene was silenced in $75 \%$ of cell lines and totally methylations and partly methylations in promotor at six cell lines by Methylation-Specific PCR (MSP). Furthermore, that study indicated that the RASSF10 gene inhibited proliferation of cancer cells by Wnt/ $\beta$-catenin signaling in vivo. Jin et al. (17) showed similar results in hepatocellular carcinoma, in which RASSF10 suppressed hepatocellular carcinoma growth by activating p53 signaling. RASSF10 induced blockage of the G2/M phase and made cancer cells sensitive to docetaxel, which indicates that RASSF10 is a resistance marker. Western blotting showed upregulation of p53 and p21 and downregulation of MDM2 and bcl-2 after overexpression of RASSF10. In our study, we treated MM cell lines with decitabine, a demethylation agent, to observe the level of RASSF10 gene 
expression. As we expected, methylation of the RASSF10 promoter was downregulated and RASSF10 gene expression upregulated after decitabine treatment.

Epigenetic aberrations play an important role in the mechanism of MM, especially DNA methylation. Hypermethylation of genes seems to be associated with the progression of monoclonal gammopathy of undetermined significance to MM and to plasma cell leukemia (18). Many studies have revealed hypermethylation of specific loci (19) and some of them are associated with poor prognosis of $\mathrm{MM}$ patients, including SPARC, BNIP3, DAPK, RARß, EGLN3, DCC, TGF $\beta R 2, C D 9, R A S D 1$ and $\mathrm{p} 16$ (20-26). These findings stress the importance of hypermethylated genes in MM. Therefore, DNMT inhibitors can target this aberrant DNA methylation in MM, such as 5-azacytidine (AZA) and decitabine. Lavelle et al. revealed that decitabine restored the expression of 16 by DNA demethylation in MM cell lines. In addition, decitabine induced G0/G1- and G2/M-phase arrest linked with p21 or p38, respectively (27). Another study showed that decitabine has potent anti-myeloma activity in vitro by depleting myeloid-derived suppressor cells in BM (28). However, use of AZA or decitabine in MM is limited because of induction of DNA damage. Recent research showed that decitabine enhanced the effect of bortezomib in an MM cell line (29). Decitabine, combined with quisinostat, a histone deacetylase inhibitor, showed increased antimyeloma effects and altered immune cell constitution, such as increased dendritic cells and naive $T$ cells, in a mouse myeloma model (30). Another study indicated that decitabine-mediated apoptosis in MM can be enhanced by combination with histone deacetylase inhibitor (31). In our study, decitabine alone reduced apoptosis by $50 \%$ in vitro, which indicates decitabine may be used in combination with other drugs.

\section{Conclusion}

The RASSF10 gene is significantly downregulated in newly diagnosed MM patients and positively correlated with overall survival. The RASSF10 gene inhibits the proliferation of myeloma cells in vitro and in vivo. We demonstrated the hypermethylation of the promoter of RASSF10, which can be modified by decitabine. To our knowledge, this is the first study to reveal the role of RASSF10 in MM and explore the effect of decitabine on this gene. However, the effect of decitabine alone in MM is not satisfactory, and combination with other types of drugs should be tried in the future.

\section{Declarations}

\section{Ethics approval and consent to participate}

The present study was approved by the Ethics Committee of Tianjin Medical University (IRB2020-WZ-075 and IRB2020-DW-04). Written informed consent was obtained for all patients.

\section{Consent for publication}

Not applicable. 
Availability of data and materials

The datasets used and/or analysed in the present study are available from the corresponding author on reasonable request.

\section{Competing interests}

The authors declare that they have no competing interests.

\section{Funding}

This work was supported by the National Natural Science Foundation of China (Grant nos. 81570106, 81770110), the anticancer major special project of Tianjin (Grant nos. 12ZCDZSY18000), the Tianjin Municipal Natural Science Foundation (Grant nos. 18JCYBJC27200, 18JCYBJC91700, 18JCQNJC80400), Tianjin Health and Family Planning Commission (Grant nos. 15KG150), the foundation of Tianjin Municipal Education Commission (2018KJ043),

\section{Authors' contributions}

RF designed the research plan and revised the manuscript. JC and HL performed the experiments, analysed the data and wrote the manuscript. ZL, QS, FJ and SY contributed to the experimental work. LL, JS and KD recorded the clinical characteristics of the patients with MM. All authors read and approved the final version of the manuscript.

\section{Acknowledgements}

We thank International Science Editing ( http://www.internationalscienceediting.com ) for editing this manuscript.

\section{Abbreviations}

MM: Multiple myeloma

BM: bone marrow

M-protein: monoclonal protein

B-ALL: B-acute lymphocytic leukemia

CLL: chronic Lymphocytic Leukemia

DNMT: DNA methyltransferase

FBS: fetal bovine serum

MACS: Magnetic-activated cell sorting 
BMMCs: BM mononuclear cells

PCR: polymerase chain reaction

PBS: Phosphate buffered saline

MOI: multiplicity of infection

HE staining: hematoxylin and eosin staining

NDMM: newly diagnosed MM

AZA: 5-azacytidine

\section{References}

1. Rajkumar SV, Dimopoulos MA, Palumbo A, Blade J, Merlini G, Mateos MV, et al. International myeloma working group updated criteria for the diagnosis of multiple myeloma. Lancet Oncol.2014;15(12):538-548.

2. Rajan AM, Kumar S. New investigational drugs with single-agent activity in multiple myeloma. Blood Cancer J.2016;6(7): e451.

3. Dimopoulos K, Gimsing P, Grunbaek K. The role of epigenetics in the biology of multiple myeloma. Blood Cancer J. 2014;4(5): e207.

4. Pawlyn C, Kaiser MF, Davies FE, Morgan GJ. Current and potential epigenetic targets in multiple myeloma. Epigenomics. 2014;6(2):215-28.

5. Wong KY, Chim CS. DNA methylation of tumor suppressor protein-coding and non-coding genes in multiple myeloma. Epigenomics. 2015;7(6):985-1001.

6. Furukawa Y, Kikuchi J. Epigenetic mechanisms of cell adhesion-mediated drug resistance in multiple myeloma. Int J Hematol. 2016;104(3):281-92.

7. Richter AM, Pfeifer GP, Dammann RH. The RASSF proteins in cancer; from epigenetic silencing to functional characterization. Biochim Biophys Acta 2009 Dec; 1796(2):114-28.

8. Sherwood V, Recino A, Jeffries A, Ward A, Chalmers AD. The N-terminal RASSF family: A new group of Ras-association-domain-containing proteins, with emerging links to cancer formation. Biochem J. 2009 Dec 23;425(2):303-11.

9. Schagdarsurengin U, Ritcher AM, Wohler C, Dammann RH. Frequent epigenetic inactivation of RASSF 10 in thyroid cancer. Epigenetics. 2009 Nov 16;4(8):571-6.

10. Hesson LB, Dunwell TL, Cooper WN, Catchpoole D, Brini AT, Chiaramonte R, et al. The novel RASSF6 and RASSF10 candidate tumour suppressor genes are frequently epigenetically inactivated in childhood leukaemias. Mol Cancer. 2009 Jul 1;8:42. 
11. Hill VK, Underhill-Day N, Krex D, Robel K, Sangan CB, Summersgill HR, et al. Epigenetic inactivation of the RASSF10 candidate tumor suppressor gene is a frequent and an early event in gliomagenesis. Oncogene. 2011 Feb 24;30(8):978-89.

12. Dansranjavin T, Wagenlehner F, Gattenloehner S, Steger $K$, Weidner W, Dammann R, et al. Epigenetic down regulation of RASSF10 and its possible clinical implication in prostate carcinoma, Prostate. 2012 Oct 1;72(14):1550-8.

13. Shinawi T, Hill V, Dagklis A, Baliakas P, Stamatopoulos K, Agathanggelou A, et al. KIBRA gene methylation is associated with unfavorable biological prognostic parameters in chronic lymphocytic leukemia. Epigenetics. 2012 Mar;7(3):211-5.

14. Moreaux J, Bruyer A, Veyrune JL, Goldschmidt H, Hose D, Klein B. DNA methylation score is predictive of myeloma cell sensitivity to 5-azacitidine. Br J Haematol. 2014 Feb;164(4):613-6.

15. Chinese Hematology Association, Chinese Society of Hematology, Chinese Myeloma CommitteeChinese Hematology Association. The guidelines for the diagnosis and management of multiple myeloma in China (2017 revision). Zhonghua Nei Ke Za Zhi. 2017 Nov 1;56(11):866-870.

16. Wei Z, Chen X, Chen J, Wang W, Xu X, Cai Q. RASSF10 is epigenetically silenced and functions as a tumor suppressor in gastric cancer. Biochem Biophys Res Commun. 2013 Mar 22;432(4):632-7.

17. Jin Y, Cao B, Zhang M, Zhan Q, Herman JG, Yu M, Guo M. RASSF10 suppresses hepatocellular carcinoma growth by activating P53 signaling and methylation of RASSF10 is a docetaxel resistant marker. Genes Cancer. 2015 May;6(5-6):231-40.

18. Walker BA, Wardell CP, Chiecchio L, Smith EM, Boyd KD, Neri A, et al. Aberrant global methylation patterns affect the molecular pathogenesis and prognosis of multiple myeloma. 2011; 117:553-562.

19. Sharma A, Heuck CJ, Fazzari MJ, Mehta J, Singhal S, Greally JM, et al. DNA methylation alterations in multiple myeloma as a model for epigenetic changes in cancer. Wiley Interdiscip. Rev. Syst. Biol. Med. 2010; 2:654-669.

20. Heller G, Schmidt WM, Ziegler B, Holzer S, Müllauer L, Bilban M, et al. Genome-wide transcriptional response to 5-aza-2'-deoxycytidine and trichostatin a in multiple myeloma cells. Cancer Res. 2008; 68:44-54.

21. Braggio E, Maiolino A, Gouveia ME, Magalhães R, Souto Filho JT, Garnica M, et al. Methylation status of nine tumor suppressor genes in multiple myeloma. J. Hematol. 2010; 91:87-96.

22. Hatzimichael E, Dasoula A, Shah R, Syed N, Papoudou-Bai A, Coley HM, et al. The prolyl-hydroxylase EGLN3 and not EGLN1 is inactivated by methylation in plasma cell neoplasia. J. Haematol. 2010; 84:47-51.

23. De Carvalho F, Colleoni GW, Almeida MS, Carvalho AL, Vettore AL. TGFbetaR2 aberrant methylation is a potential prognostic marker and therapeutic target in multiple myeloma. J. Cancer. 2009; 125:1985-1991.

24. De Bruyne E, Bos TJ, Asosingh K, Vande Broek I, Menu E, Van Valckenborgh E, et al. Epigenetic silencing of the tetraspanin CD9 during disease progression in multiple myeloma cells and correlation with survival. Cancer Res. 2008; 14:2918-2926. 
25. Stanganelli C, Arbelbide J, Fantl DB, Corrado C, Slavutsky I. DNA methylation analysis of tumor suppressor genes in monoclonal gammopathy of undetermined significance. Ann Hematol. 2010; 89:191-199.

26. Nojima M, Maruyama R, Yasui $H$, Suzuki $H$, Maruyama $Y$, Tarasawa I, et al. Genomic screening for genes silenced by DNA methylation revealed an association between RASD1 inactivation and dexamethasone resistance in multiple myeloma. Clin Cancer Res. 2009; 15:4356-4364.

27. Lavelle D, DeSimone J, Hankewych M, Kousnetzova T, Chen YH. Decitabine induces cell cycle arrest at the G1 phase via p21(WAF1) and the G2/M phase via the p38 MAP kinase pathway. Leuk Res. 2003; 27:999-1007.

28. Zhou J, Shen Q, Lin H, Hu L, Li G, Zhang X. Decitabine shows potent anti-myeloma activity by depleting monocytic myeloid-derived suppressor cells in the myeloma microenvironment. J Cancer Res Clin Oncol. 2018 Nov 13. doi: 10.1007/s00432-018-2790-6.

29. De Beck L, Melhaoui S, De Veirman K, Menu E, De Bruyne E, Vanderkerken K, et al.Epigenetic treatment of multiple myeloma mediates tumor intrinsic and extrinsic immunomodulatory effects. Oncoimmunology. 2018 Jul 23;7(10): e1484981. doi: 10.1080/2162402X.2018.1484981

30. Cao Y, Qiu GQ, Wu HQ, Wang ZL, Lin Y, Wu W, Xie XB, Gu WY.Decitabine enhances bortezomib treatment in RPMI 8226 multiple myeloma cells. Mol Med Rep. 2016 Oct;14(4):3469-75. doi: 10.3892/mmr.2016.5658.

31. Maes K, De Smedt E, Lemaire M, De Raeve H, Menu E, Van Valckenborgh E,.The role of DNA damage and repair in decitabine-mediated apoptosis in multiple myeloma. Oncotarget. 2014 May 30;5(10):3115-29.

32. Maes K, Menu E, Van Valckenborgh E, Van Riet I, Vanderkerken K, De Bruyne E. Epigenetic Modulating Agents as a New Therapeutic Approach in Multiple Myeloma. 2013; 5:430-461.

\section{Tables}

Table1. The clinical characteristics the patients of newly diagnosed and remission MM 


\begin{tabular}{|c|c|c|c|}
\hline Clinical features & Newly diagnosed MM & Remission MM & Controls \\
\hline $\mathbf{N}$ & 30 & 17 & 19 \\
\hline Median age[range[ & $66(44-81)$ & $60(45-75)$ & $37(19-69)$ \\
\hline \multicolumn{4}{|l|}{ Gender $\square N, \% \square$} \\
\hline Male & $17(56.7)$ & $7(41.2)$ & $9(47.4)$ \\
\hline Female & $13(43.3)$ & $10(58.8)$ & $10(52.6)$ \\
\hline \multicolumn{4}{|l|}{ Subtpye } \\
\hline Light chain MM (N, \%) & $7(23.3)$ & $2(11.8)$ & \\
\hline $\operatorname{IgG~k~}(\mathrm{N}, \%)$ & $10(33.3)$ & $8(47.1)$ & \\
\hline $\operatorname{IgG} \lambda(N, \%)$ & $7(23.3)$ & $1(5.88)$ & \\
\hline $\operatorname{IgA~} \mathrm{k}(\mathrm{N}, \%)$ & $2(6.67)$ & $1(5.88)$ & \\
\hline $\operatorname{IgA} \lambda(N, \%)$ & $4(13.3)$ & $1(5.88)$ & \\
\hline Non secreting type(N, \%) & 0 & $4(23.5)$ & \\
\hline \multicolumn{4}{|l|}{ ISS stage (N, \%) } \\
\hline प & $3(10.0)$ & $7(41.2)$ & \\
\hline$\square$ & $15(50.0)$ & $5(29.4)$ & \\
\hline प & $12(40.0)$ & $5(29.4)$ & \\
\hline High-risk Fish(N, \%) & $11(36.7)$ & $5(29.4)$ & \\
\hline
\end{tabular}

MM: multiple myeloma; N: number;

Table 2. The cell proliferation rates of MM cell lines after overexpression of RASSF10.

\begin{tabular}{ccccc}
\hline & \multicolumn{4}{c}{ Cell proliferation (OD value) } \\
\cline { 2 - 5 } & $24 \mathrm{~h}$ & $48 \mathrm{~h}$ & $72 \mathrm{~h}$ & $96 \mathrm{~h}$ \\
\hline RPMI-8266/Cnt & $0.94 \pm 0.01$ & $1.67 \pm 0.06$ & $1.81 \pm 0.06$ & $2.17 \pm 0.08$ \\
\hline RPMI-8266/RASSF10 & $0.98 \pm 0.03$ & $1.27 \pm 0.08^{*}$ & $1.28 \pm 0.05^{*}$ & $1.35 \pm 0.02^{*}$ \\
\hline OPM-2/Cnt & $1.34 \pm 0.03$ & $1.68 \pm 0.10$ & $1.84 \pm 0.03$ & $2.38 \pm 0.05$ \\
\hline OPM-2/RASSF10 & $1.34 \pm 0.03$ & $1.50 \pm 0.04^{*}$ & $1.62 \pm 0.08^{*}$ & $1.99 \pm 0.01^{*}$ \\
\hline
\end{tabular}

${ }^{*}$ compared with Cnt group, $p<0.01$.

Table 3. The cell apoptosis rates of MM cell lines after overexpression of RASSF10.

\begin{tabular}{ccccc}
\hline & \multicolumn{4}{c}{ Apoptosis rate (\%) } \\
\cline { 2 - 5 } & $24 \mathrm{~h}$ & $48 \mathrm{~h}$ & $72 \mathrm{~h}$ & $96 \mathrm{~h}$ \\
\hline RPMI-8266/Cnt & $27.37 \pm 2.67$ & $39.42 \pm 3.38$ & $40.97 \pm 1.73$ & $47.99 \pm 2.62$ \\
\hline RPMI-8266/RASSF10 & $41.02 \pm 4.63^{*}$ & $44.76 \pm 5.07$ & $52.31 \pm 1.23^{*}$ & $63.96 \pm 8.19^{*}$ \\
\hline OPM-2/Cnt & $17.60 \pm 0.99$ & $19.12 \pm 2.23$ & $20.25 \pm 1.14$ & $24.70 \pm 0.78$ \\
\hline OPM-2/RASSF10 & $32.47 \pm 2.55^{*}$ & $35.26 \pm 7.03^{*}$ & $33.38 \pm 1.72^{*}$ & $44.04 \pm 1.72^{*}$ \\
\hline
\end{tabular}


*compared with Cnt group, $p<0.01$.

\section{Figures}
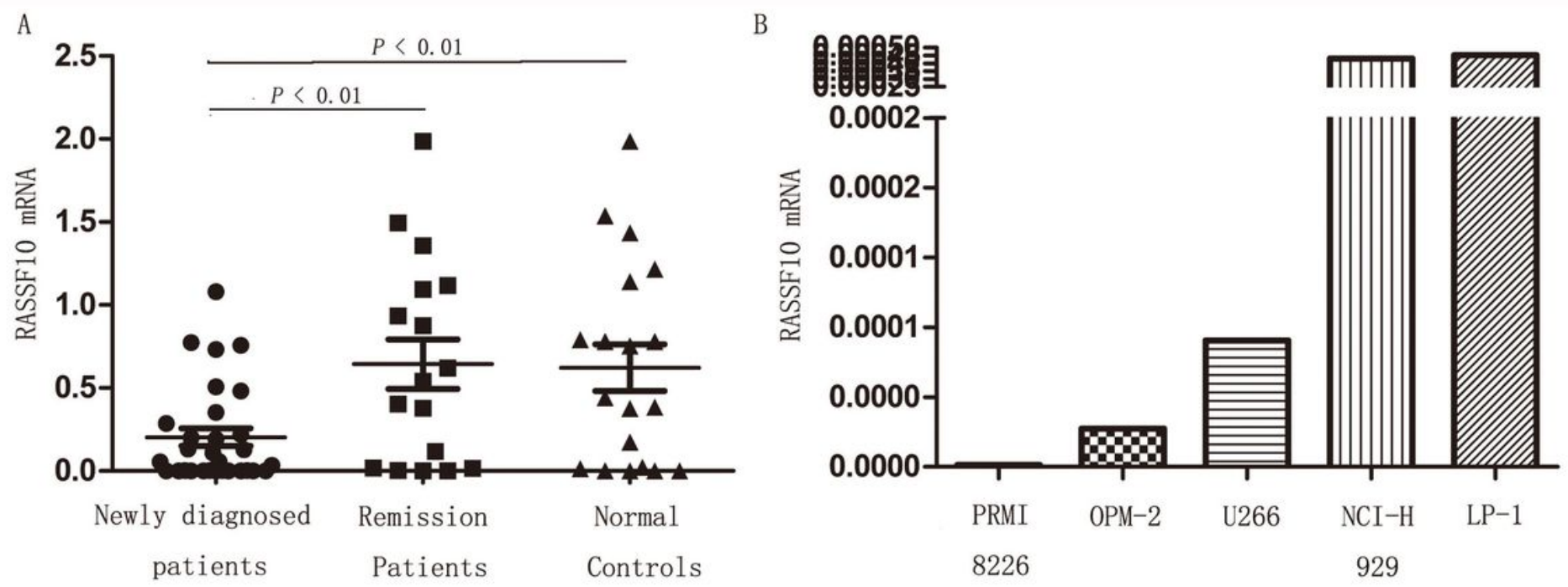

C

D

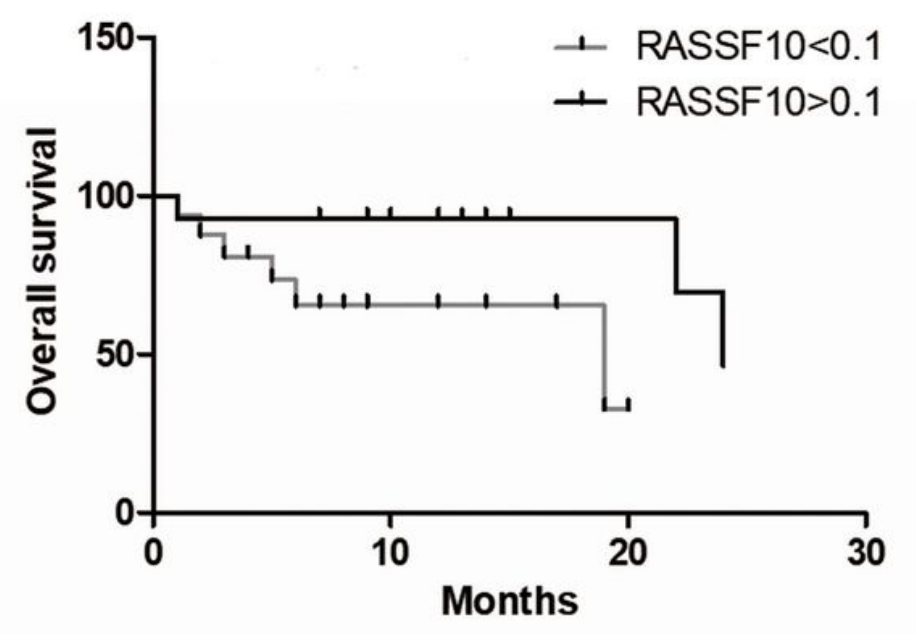

Figure 1

Expression of RASSF10 mRNA in MM patients and cell lines. A. RASSF10 mRNA expression was significantly lower in newly diagnosed MM patients. B. RASSF10 mRNA expression in different MM cell lines. C. Protein expression of the RASSF10, MM patients, normal control and MM cell lines RPMI-8226, OPM-2 and U266 as detected by western blotting. D. Overall survival of newly diagnosed MM patients with different levels of RASSF10 expression. 

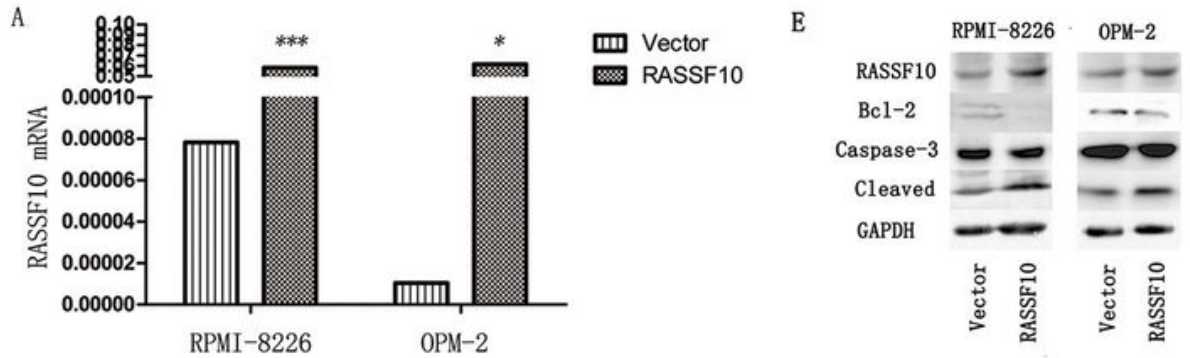

B1

RPMI-8226

B2

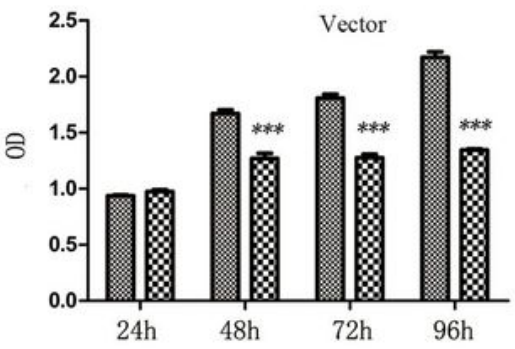

RASSF10

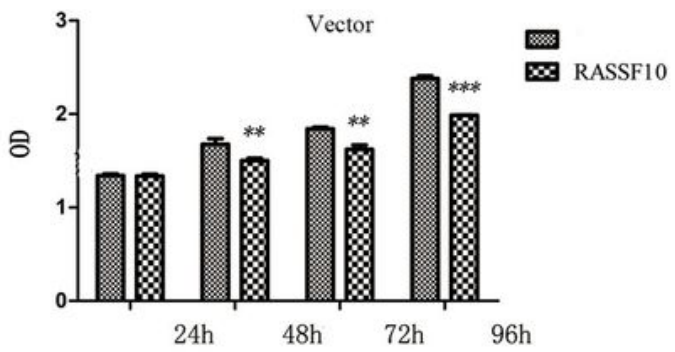

$\mathrm{C} 1$
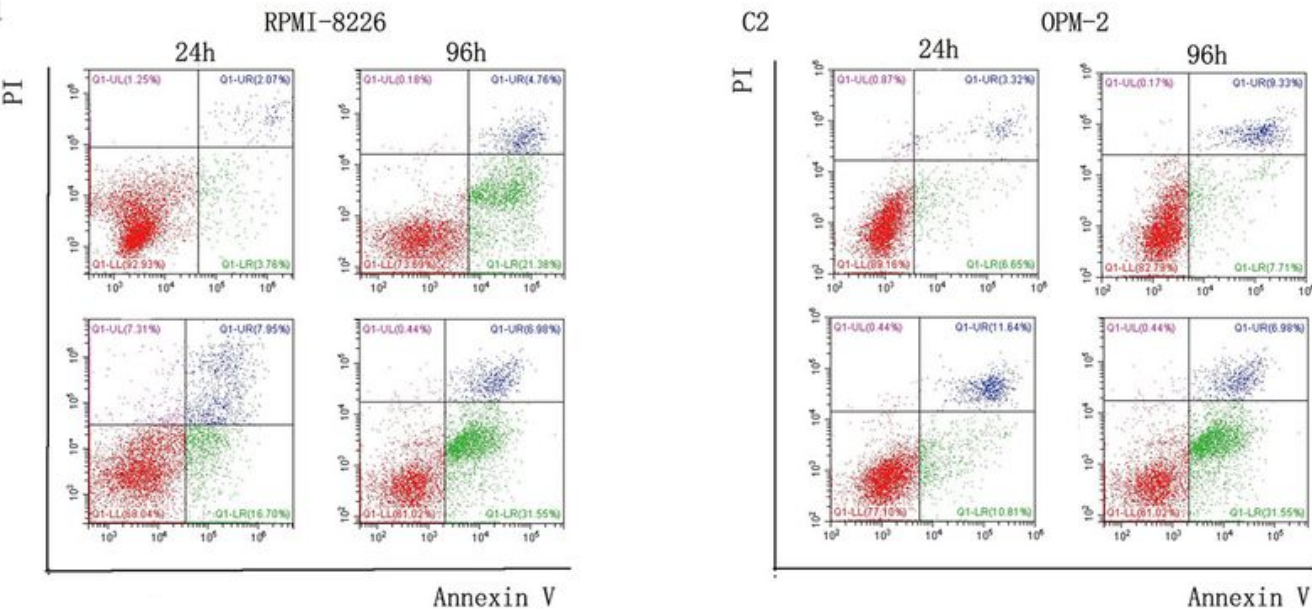

D1

RPMI-8226

D2

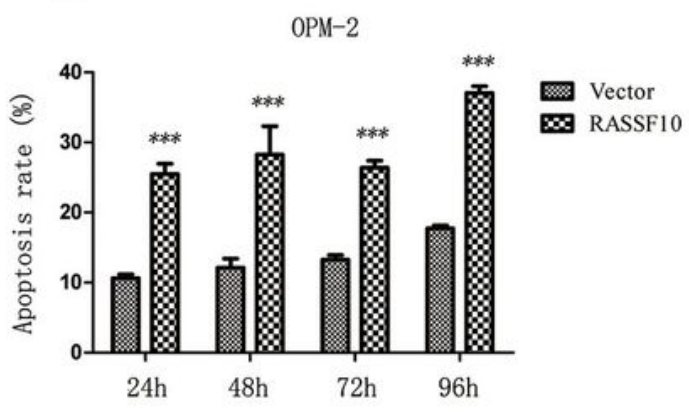

Figure 2

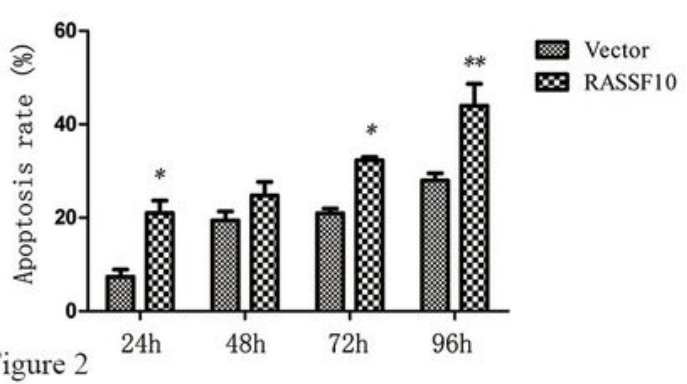

Figure 2

Proliferation of MM cell lines was inhibited after RASSF10 overexpression. A. RASSF10 expression upregulated after transfection in RPMI-8226 and OPM-2 cells. B. Cell survival was inhibited after RASSF10 overexpression in RPMI-8226 (B1) and OPM-2 (B2) cells. C. Apoptosis was detected by flow cytometry using annexin V and PI in RASSF10 overexpression in RPMI-8226 (C1) and OPM-2 (C2) cells. D. Apoptosis of cell lines increased after RASSF10 overexpression in RPMI-8226 (D1) and OPM-2 (D2) cells. 
E. Protein expression of RASSF10, apoptosis-related protein the cleaved caspase-3 and apoptosis-related protein bcl-2 as detected by western blotting. ${ }^{*} p<0.05$, ${ }^{\star *} p<0.01,{ }^{* \star *} p<0.001$.

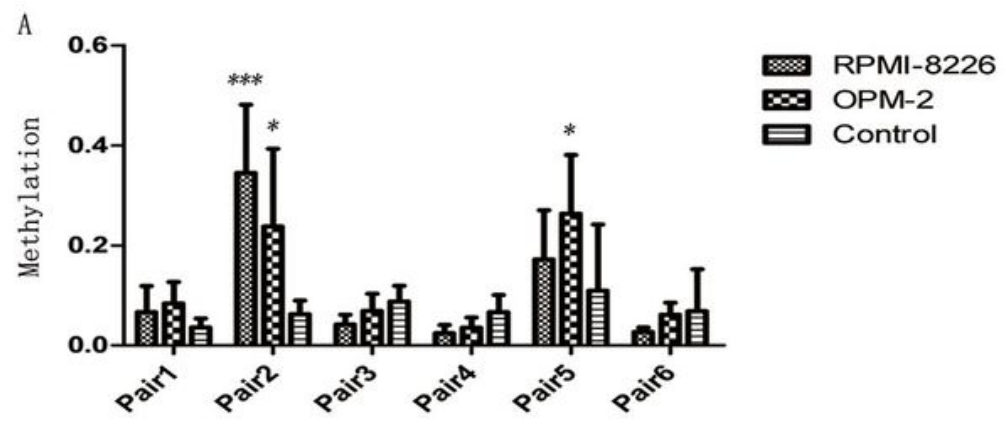

B1

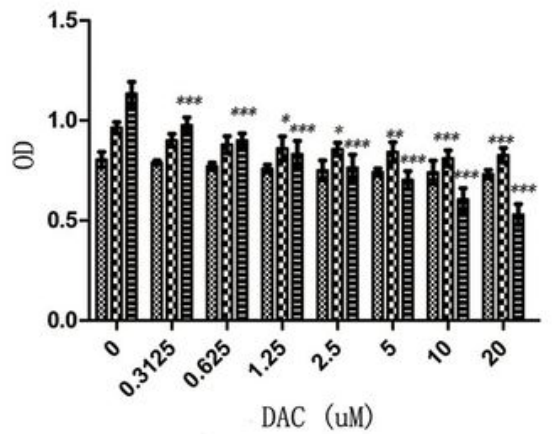

C1

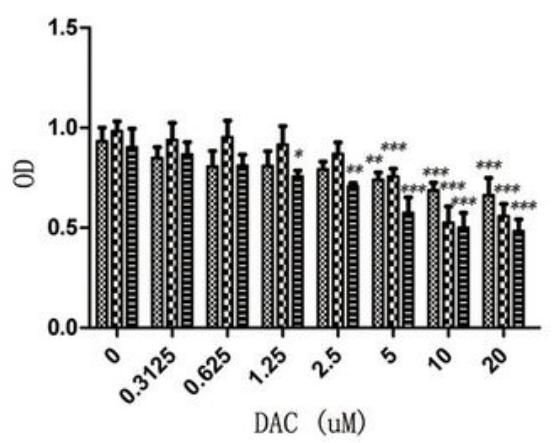

D

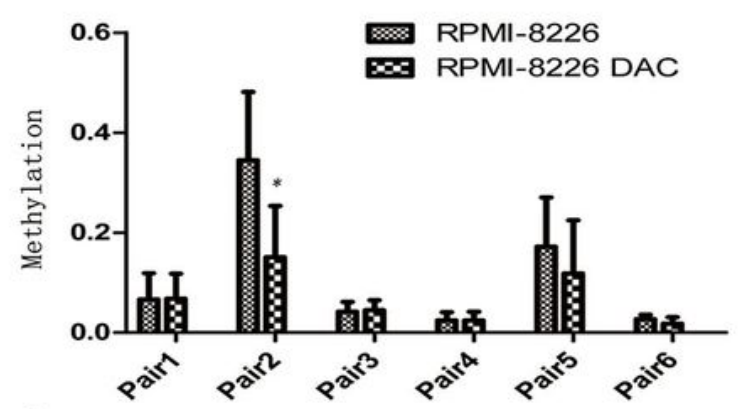

B2

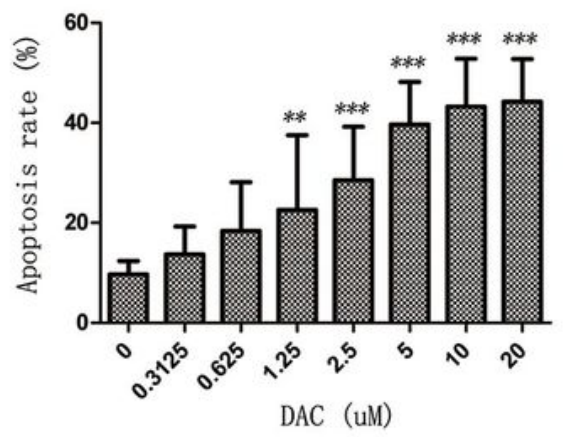

$\mathrm{C} 2$

OPM-2
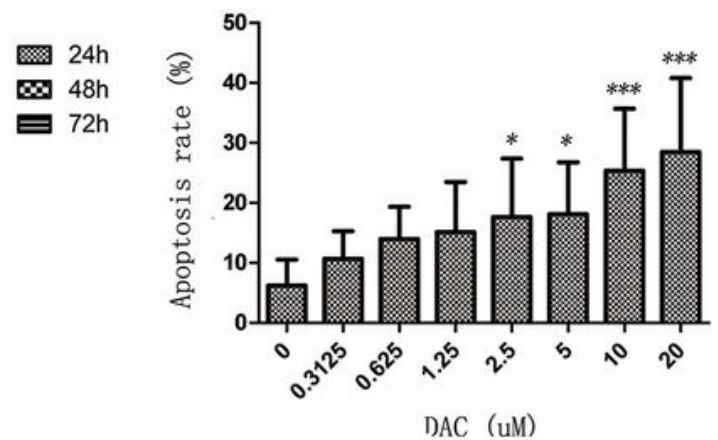

E

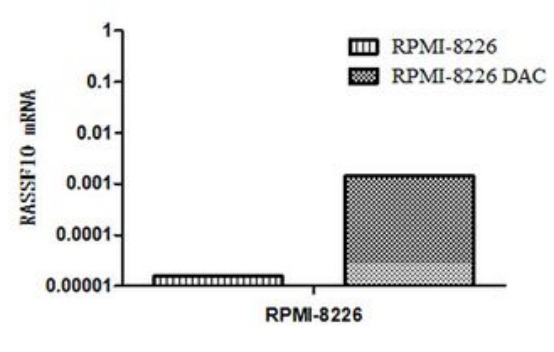

Figure 3

Figure 3

MM cell lines treated with decitabine and methylation of RASSF10 promoter downregulated after decitabine treatment. A. Methylation of the RASSF10 gene promoter in RPMI-8226 and OPM-2 cells detected by bisulfite sequencing. B. Survival of RPMI-8226 cells after decitabine treatment for 24,48 and 
$72 \mathrm{~h}$ (B1). Apoptosis of RPMI-8226 cells after decitabine treatment for $72 \mathrm{~h}$ (B2). C. Survival of OPM-2 cells after decitabine treatment for 24,48 and $72 \mathrm{~h}(\mathrm{C} 1)$. Apoptosis of OPM-2 cells after decitabine treatment for $72 \mathrm{~h}$ (C2). D. Methylation of the RASSF10 gene promoter in RPMI-8226 cells detected after decitabine treatment $(2.5 \mathrm{\square M})$ for $72 \mathrm{~h} .{ }^{*} \mathrm{p}<0.05,{ }^{\star \star} \mathrm{p}<0.01,{ }^{\star \star *} \mathrm{p}<0.001$.

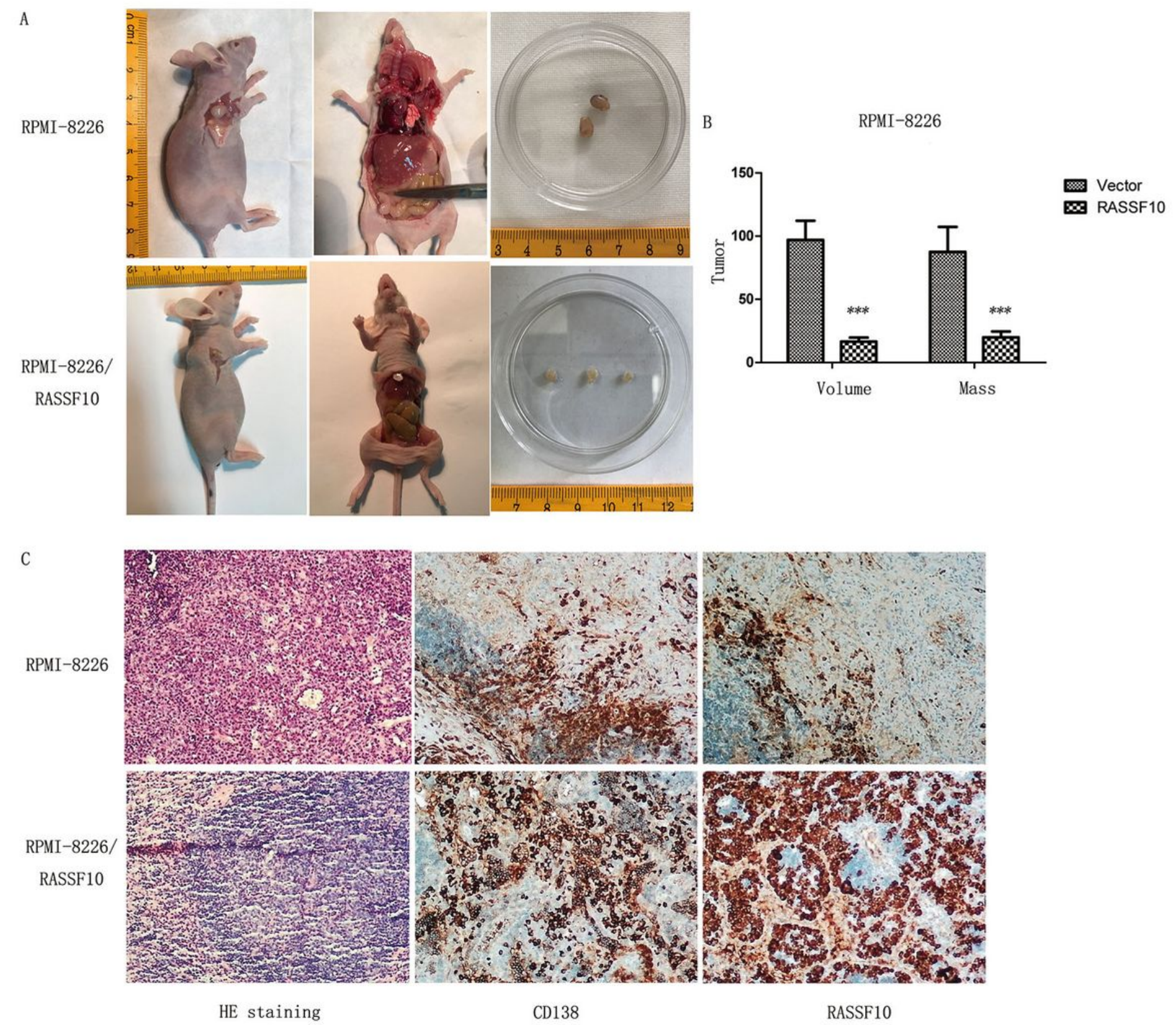

Figure 4

Figure 4

Role of RASSF10 gene overexpression in MM animal model. A. Subcutaneous tumor, lymph nodes, liver and spleen were observed at 21 days after RPMI-8226/Vector and RPMI-8226/RASSF10 injection. B. Tumor volume and mass of RPMI-8226/RASSF10 were significantly reduced compared with RPMI8226/Vector. C. HE staining, CD138 and RASSF10 staining of subcutaneous tumor in RPMI-8226/Vector and RPMI-8226/RASSF10 mice. ${ }^{* \star} \mathrm{p}<0.001$. 